\section{A case of follicular lymphoma complicated with mesenteric panniculitis}

Yotaro Tamai,, Osamu Imataki, ${ }^{2}$
Ichiro Ito, ${ }^{3}$ Kimihiro Kawakami'

'Division of Hematology \& Stem Cell

Transplantation, Shizuoka Cancer Center, Shizuoka, Japan; ${ }^{2}$ Division of

Hematology, Department of Internal

Medicine, Faculty of Medicine, Kagawa

University, Kagawa, Japan; ${ }^{3}$ Division of

Pathology, Shizuoka Cancer Center,

Shizuoka, Japan

\section{Abstract}

Mesenteric panniculitis (MP) is a rare disease occasionally complicated with lymphoma. A 55-year old female presented with MP accompanied by malignant lymphoma. This patient was first treated for follicular lymphoma and subsequently for panniculitis. After 6 courses of R-CHOP chemotherapy, the treatment response was partial. An additional course of salvage chemotherapy led to a complete response. Since the mesenteric mass progressed simultaneously with the regression of other lymphoma lesions, we performed a biopsy of the mesenteric mass and pathologically confirmed an MP lesion without lymphoma. Subsequent high-dose chemotherapy led to CR and the MP lesion remained stable. In the present case, MP progressed with chemotherapy. We concluded that mesenteric lesions suspected of progressing or recurring should be diagnosed pathologically even if asymptomatic.

\section{Introduction}

An extensive involvement of mesenteric fat tissue of the small bowel with chronic non-specific inflammatory disease, first reported by Jura et al. in $1924^{1}$ was described, detailed, and named as mesenteric panniculitis (MP) by Ogden et al. in $1960 .{ }^{2} \mathrm{MP}$ is always discussed with pathological findings concerning its etiology and 3 components of histology, fibrosis, chronic inflammation, and fat necrosis, suggesting various mechanisms of disease (called sclerotic mesenteritis, mesenteric panniculitis, and mesenteric lipodystrophy, respectively) ${ }^{3}$ Histologically, MP lesion is characterized by the infiltration of mesenteric fat tissue by inflammatory cells, mainly consisting of lymphocytes and plasmacytes, along with a mixture of fat necrosis and fibrosis. ${ }^{3}$ Although the diagnosis of MP before biopsy is primarily based on guesswork, computed tomography (CT) findings are helpful for cases in which mesentery biopsy is problematic. ${ }^{4}$ The clinical course of MP is usually benign, and spontaneous remission is quite common without specific treatment. $^{5}$

The pathogenesis of MP remains obscure and many associated diseases are known, including mechanical stimulation such as surgery or trauma, infections, vascular diseases, and malignant and non-malignant neoplasms. ${ }^{5}$ The occurrence rate of lymphoma as an underlying disease is $15 \%$ or more, and no malignant involvement of the mesentery was diagnosed in almost all cases. ${ }^{5}$ In a case series of MP accompanied with malignant lymphoma, Kipfer et al. (8 cases) suggested that mesenteritis was a non-specific response to underlying lymphoma. ${ }^{5}$ Other reports introduced MP incidence during the clinical follow-up course
Correspondence: Osamu Imataki, Division of Hematology, Department of Internal Medicine, Faculty of Medicine, Kagawa University,1750-1 Ikenobe, Miki-cho, Kita-gun, Kagawa, 761-0793, Japan. E-mail: oima@med.kagawa-u.ac.jp

Key words: follicular lymphoma, mesenteric panniculitis, autologous peripheral blood stem cell transplantation.

Received for publication: 11 July 2009. Revision received: 3 October 2009.

Accepted for publication: 5 October 2009.

This work is licensed under a Creative Commons Attribution 3.0 License (by-nc 3.0).

(C) Copyright Y. Tamai et al., 2009

Licensee PAGEPress, Italy

Hematology Reviews 2009; 1:e17

doi:10.4081/hr.2009.e17 (a)
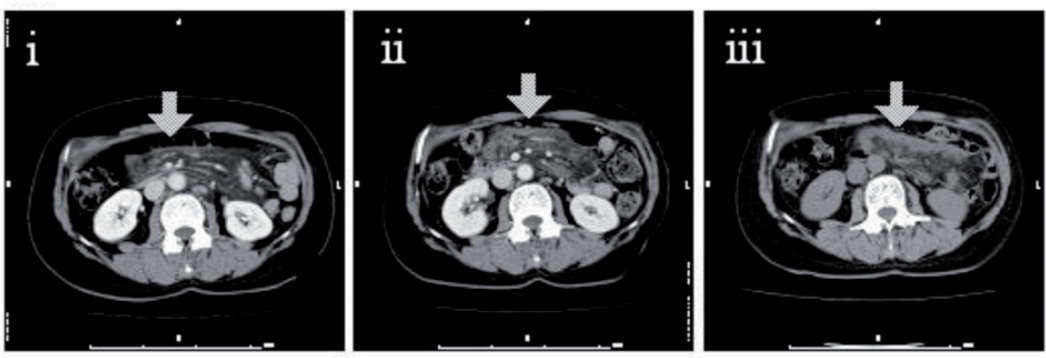

(b)
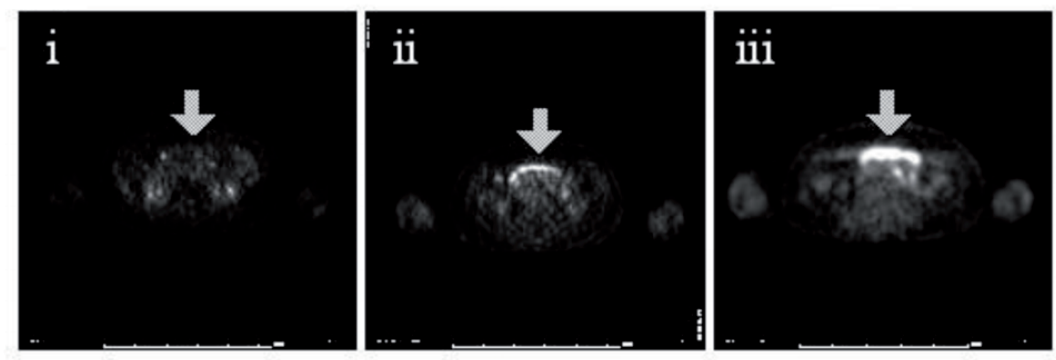

(c)
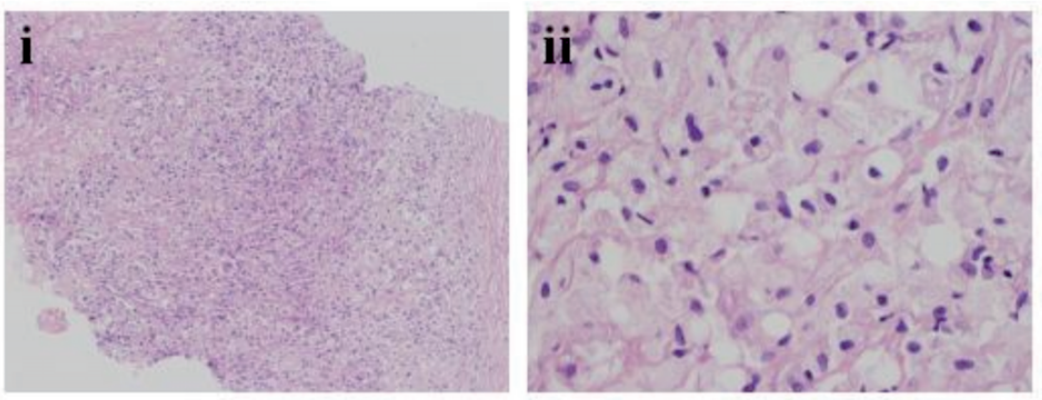

Figure 1. Imaging and pathologic evaluation of mesenteric panniculitis. During treatment and before SCT, we evaluated the clinical course of the mesenteric mass by CT (A) and 18F-FDG-PET (B). (A) Clinical course of mesenteric lesion evaluated by CT imaging. Mesenteric hypertrophy before treatment reduced during chemotherapy (i), after salvage chemotherapy (ii), and before PBSCT (iii). Arrows indicate hypertrophic mesenterium. (B) Clinical course of mesenteric lesion evaluated by FDG-PET. An accumulation positioned at the mesenterium detected by FDG-PET (i) was more intensified after salvage chemotherapy (ii) and did not improve before PBSCT (iii). Arrows indicate an increased accumulation in accordance with the enlarged mesenterium. (C) Pathological findings of biopsied mesenteric mass. Fatty necrosis surrounded by foamy macrophages and reactive lymph adenopathy was characteristic of the mesenterium. Immunohistological staining failed to detect CD20, CD10, CD79a, and bcl-2, with no indications of malignant lymphoma: (i) H-E stain (x 400), (ii) H-E stain (x 1000). 
of lymphoma. Ogden (2 cases) ${ }^{6}$ and PerezFerriols (1 case $)^{7}$ both reported on the association of these 2 diseases.

\section{Case Report}

A 55-year old woman presented with right cervical lymph node swelling in July, 2004, followed by inguinal lymph node swelling a few weeks later. She was admitted to our hospital in August, 2004. The results of the blood and bone marrow examinations performed at that time are shown in Table 1. A lymph node biopsy of the inguinal area revealed grade 2 follicular lymphoma. CT images of her neck, thorax and abdomen, and FDG-PET revealed stage IV disease. She was diagnosed as having stage IV lymphoma with femoral bone involvement, as confirmed by femoral MRI. Her follicular lymphoma international prognostic index (FLIPI) was intermediate (stage and lymph-node lesions). IgH-BCL rearrangement (FISH) was detected in her lymph nodes and bone marrow cells, although neither morphological abnormality nor apparent involvement of lymphoma cells were revealed. We did not examine her for IgH-BCL rearrangement of peripheral blood. B symptoms were absent; however, since she complained of bone pain and it was progressive, we decided to treat her with chemotherapy. R-CHOP chemotherapy consisted of rituximab $\left(375 \mathrm{mg} / \mathrm{m}^{2}\right.$, day 0$)$, cyclophosphamide (750 mg/m² day 1$)$, doxorubicin $\left(50 \mathrm{mg} / \mathrm{m}^{2}\right.$, day $1)$, vincristine $\left(1.4 \mathrm{mg} / \mathrm{m}^{2}\right.$, day 1$)$, and prednisolone (100 mg/kg body weight, day $1-5)$, injected tri-weekly until 6 courses were completed. At this point only a partial response (PR) was observed through objective radiological evaluations (for nodal masses FDG-PET was negative and more than 50\% regression was observed on CT). After R-CHOP, 2 courses at a 3week interval of the ACES regimen (cytarabine $2,000 \mathrm{mg} / \mathrm{m}^{2}$ day 5 , carboplatin $100 \mathrm{mg} / \mathrm{m}^{2}$ day 1 4, etoposide $80 \mathrm{mg} / \mathrm{m}^{2}$ days $1-4$, methylprednisolone $500 \mathrm{mg} /$ body days 1-5) were used as salvage chemotherapy, after which her disease was considered to be in CR (IgH-BCL rearrangement in her lymph nodes and bone marrow became negative). At the same time, a contrastenhanced peritoneal mass appeared on CT imaging (Figure 1A) and FDG-PET (Figure 1B). We identified the pathological etiology of the peritoneal lesion proceeding to the peripheral blood stem cell transplantation (PBSCT) by CTguided biopsy, which revealed mesenteric panniculitis (MP) not concomitant with the lymphoma lesion (Figure 1C). Her MP did not produce symptoms through her treatment course. However, the bone lesion recurred in June, 2005, when she received PBSCT. She has maintained CR with no progression of the MP mass for more than three years.

Table 1. Laboratory data on admission. The profile of the peripheral blood cell count was within the normal range. Biochemical data showed a normal LDH level and no organ disorders. Cytogram of bone marrow revealed no obvious morphological changes and the involvement of lymphoma cells was not detected.

\section{Component of blood cell \\ White blood cells \\ Granulocytes \\ Eosinophils \\ Basaophils \\ Monocytes \\ Lymphocytes \\ Red blood cells \\ Hemoglobin \\ Hematocrit \\ Platelets}

\section{Biochemistry}

Total protein

Albumin

Total billirubin

Glutamic pyruvic transaminase

Lactate dehydrogenase

Alkaline phosphatase

Blood urea nitrogen

Creatinine

Urine acid

Natrium

Potassium

Chloride

\section{Coagulation}

Prothrombin time

Activated partial thromboplastin time

International normalized ratio

Fibrinogen

Bone marrow

Number of nuclear cells

Megakaryocytes

Myeloid/erythroid ratio

Blasts

Promyelocytes

\author{
$9100 / \mu \mathrm{L}(3500 \sim 9100)$ \\ $69 \%(32 \sim 79)$ \\ $1 \%(0 \sim 6)$ \\ $2 \%(0 \sim 2)$ \\ $3 \%(0 \sim 8)$ \\ $25 \%(18 \sim 59)$ \\ $415 \times 10^{4} / \mu \mathrm{L}(376 \sim 500)$ \\ $12.6 \mathrm{~g} / \mathrm{dL} 11.3 \sim 15.2)$ \\ $37.4 \%(33.4 \sim 44.9)$ \\ $23.1 \times 10^{4} / \mu \mathrm{L}(13.0 \sim 36.9)$
}

$7.7 \mathrm{~g} / \mathrm{dL}(6.7 \sim 8.3)$

$4.6 \mathrm{~g} / \mathrm{dL}(4.0 \sim 5.0)$

$0.4 \mathrm{mg} / \mathrm{dL}(0.3 \sim 1.2)$

$19 \mathrm{IU} / \mathrm{L}(5 \sim 40)$

$195 \mathrm{IU} / \mathrm{L}(115 \sim 245)$

259 IU/L (115 359)

$15.9 \mathrm{mg} / \mathrm{dL}(8.0 \sim 22.0)$

$0.6 \mathrm{mg} / \mathrm{dL}(0.47 \sim 0.79)$

$3.8 \mathrm{mg} / \mathrm{dL}(2.5 \sim 7.0)$

$142 \mathrm{mEq} / \mathrm{L}(136 \sim 147)$

$4.1 \mathrm{mEq} / \mathrm{L}(3.6 \sim 5.0)$

$106 \mathrm{mEq} / \mathrm{L}(98 \sim 109)$

$97 \%(70 \sim 130)$
$28.5 \mathrm{sec} \quad(24.3 \sim 36.0)$
$1.02(0.85 \sim 1.15)$
$274 \mathrm{mg} / \mathrm{dL}(150 \sim 400)$

$9.4 \times 10^{4} / \mu \mathrm{L}(10 \sim 20)$

$43.8 \times 10^{4} / \mu \mathrm{L}(50 \sim 150)$

$2.36(2 \sim 4)$

$0.2 \%(1.0 \sim 1.5)$

$0.6 \%(2.0 \sim 6.5)$

\section{Discussion}

Mesenteric panniculitis is a lipodystrophy characterized by non-specific inflammatory disease first reported by Ogden et al. ${ }^{2}$ Emory et al. reviewed 84 cases of MP pathologically and documented that a mixed histology of 3 components (fibrosis, chronic inflammation, and fat necrosis) existed in the lesions, which could be classified according to the dominant pathological changes, i.e. mesenteric lipodystrophy (ML) type, mesenteric panniculitis (MP) type, and sclerosing mesenteritis (SM) type. However, not all cases can be definitively categorized in these 3 subtypes because the histologically dominant subtype is often too varied to define as ML, MP, or SM, respectively. ${ }^{3}$ Due to this pathological variation and the various underlying diseases of MP, the etiology of this disease is thought to be a single pathology with various causes including immunological reactions, malignancies, infection, physical stimulation, or traumatic disorders.
Past retrospective analysis reported that, in general, MP occurs in patients in their $60 \mathrm{~s}$ (median age of occurrence), has slightly higher incidence in men, is accompanied by abdominal pain, and is often associated with malignant lymphoma. ${ }^{8,9}$ Generally, isolated MP does not need to be treated. ${ }^{4}$ Kipfer et al. reported that 8 among 53 reviewed cases of MP with malignant lymphoma led to complications related to MP. 5 They could find no pathological involvement of lymphoma in the panniculitis lesion in any case. In 3 of the 8 cases, MP existed with malignant lymphoma concomitantly. In 2 other patients, the lymphoma lesion was found two or three months later. Another 3 patients were diagnosed as having malignant lymphoma occurring in a different area from the MP lesion. These findings suggest that MP can precede or proceed to lymphoma. However, no panniculitis has been revealed in lymphoma lesions by biopsy, and no obvious association has been reported until now. In our case, the mesenteric lesion progressed and was detected by FDG-PET imaging during 
chemotherapy, which reduced to remission the lymphoma lesions (lymph nodes and femoral bone head). This discrepant course made it problematic for physicians to decide on a treatment plan. If we had regarded the mesenteric mass in our patient as refractory lymphoma, we would have had to administer a salvage regimen. If the mesenteric mass had been benign, we could have continued the existing regimen. A biopsy confirmed the remission of her lymphoma and resolved this problem. Autologous PBSCT brought about a durable CR in the patient. The MP lesion regressed after the planned treatments, with no recurrence for three years. Therefore, we recognized that her MP was chemotherapy-related panniculitis, since panniculitis is thought to be brought about by physical or chemical stimulation.

Although FDG-PET was an effective and powerful tool for the staging, localization, and response assessment of malignant lymphoma, the differential diagnosis for lymphoma lesions with physiological accumulation or inflammatory change is sometimes difficult. ${ }^{10,11}$ Though FDG-PET/CT fusion imaging is more efficient for diagnosis of the active lymphoma lesion, ${ }^{11}$ a pathological diagnosis via biopsy is essential for cases in which a false positive is suspected. Zissin et al. reviewed 19 oncological patients with $\mathrm{MP}^{12}$ and reported that FDG-PET had high specificity (100\%) among 11 FDG-
PET-negative subjects and $88 \%$ sensitivity (7 out of 8 subjects). We concluded that mesenteric lesions suspected of progressing or recurring with positive uptake with FDG-PET should be diagnosed pathologically even if asymptomatic.

\section{References}

1. Jura V. Sulla mesenterite retrattile e sclerosante. Policlinico (sez. prat) 1924;31: 575-8

2. Ogden WW 2nd, Bradburn DM, Rrives JD. Panniculitis of the mesentery. Ann Surg 1960;151:659-68.

3. Emory TS, Monihan JM, Car NJ, Sobin LH. Sclerosing mesenteritis, mesenteric panniculitis and mesenteric lipodystrophy: a single entity? Am J Surg Pathol 1997;21: 392-8.

4. Horton KM, Lawler LP, Fishman EK. CT Findings in sclerosing mesenteritis (panniculitis): spectrum of disease. Radiographics 2003;23:1561-7.

5. Kipfer RE, Moertel CG, Dahlin DC. Mesenteric lypodystrophy. Ann Intern Med 1974;80: 582-8.

6. Ogden WW, Bradburn DM, Rives JD. Mesenteri Panniculitis: review of 27 cases.
Ann Surg 1965; 161:864-75.

7. Perez-Ferriols, Feber-Bosch. Primary soft tissue lymphoma associated with mesenteric lipodystrophy. Am J Dermato-pathol 1993;15:363-7.

8. Delgado Plasencia L, Rodríguez Ballester L, López-Tomassetti Fernández EM, et al. Mesenteric panniculitis: experience in our center. Rev Esp Enferm Dig 2007;99:291-7.

9. Béchade D, Durand X, Desramé J, et al. Etiologic spectrum of mesenteric panniculitis: report of 7 cases. Rev Med Interne 2007;28:289-95.

10. Juweid ME, Stroobants S, Hoekstra OS, et al. Imaging Subcommittee of International Harmonization Project in Lymphoma. Use of positron emission tomography for response assessment of lymphoma: consensus of the Imaging Subcommittee of International Harmonization Project in Lymphoma. J Clin Oncol 2007;25:571-8.

11. Janikova A, Bolcak K, Pavlik T, et al. Value of [18F] fluorodeoxyglucose positron emission tomography in the management of follicular lymphoma: the end of a dilemma? Clin Lymphoma Myeloma 2008;8:28793.

12. Zissin R, Metser U, Hain D, et al. Mesenteric panniculitis in oncologic patients: PET-CT findings. Br J Radiol 2006;79:37-43. 